\title{
Synthetic organs for transplant and bio-mimic reactors for process intensification using nano-structured micro-porous materials
}

\author{
E. M. Akay ${ }^{1}$, Z. Okumus ${ }^{2}$, O. S. Yildirim ${ }^{3,4}$, M. A. Bokhari ${ }^{4,5}$ \\ \& G. Akay ${ }^{5,6}$ \\ ${ }^{I}$ North Middlesex University Hospital, University College London, UK \\ ${ }^{2}$ Veterinary Faculty, Ataturk University, Turkey \\ ${ }^{3}$ Medical Faculty, Department of Orthopaedic, Ataturk University, \\ Turkey \\ ${ }^{4}$ Medical Faculty (Orthopaedics), Newcastle University, UK \\ ${ }^{5}$ Process Intensification and Miniaturization Centre, \\ School of Chemical Engineering and Advanced Materials, \\ Newcastle University, UK \\ ${ }^{6}$ The Institute of Stem Cell Biology and Regenerative Medicine, \\ Newcastle University, $U K$
}

\begin{abstract}
In order to achieve Process Intensification through monolithic micro-reactors in bio- and chemical technology we examined the processing strategy in the human body, taking the liver as a typical organ. The macro- and micro-architectural structure can be mimicked by using nano-structured micro-porous polymers which were used as support in tissue engineering and bioprocess intensification. It was shown that these materials could be used as bone transplant and they are integrated into the body. Their integration could be predicted through in vitro experiments. Metallic versions of these structures with a hierarchy of pore size were produced in order to obtain catalytic bio- or chemical reactors operating at high temperature and/or pressure.

Keywords: bio-mimic materials, BioProcess Intensification, ChemicalProcess Intensification, nano-structured micro-porous materials.
\end{abstract}




\section{Introduction}

\subsection{Process intensification}

Process Intensification (PI) emerged as a novel process design philosophy in which the processing volume is deliberately reduced while the processing vectors (conditions) were enhanced in order to achieve the same levels of production rate. This resulted in the reduction of chemical plant/unit operation capital and processing costs [1]. Process Intensification is achieved via two routes [1]. Firstly, PI is a direct result of the imposition of enhanced and uniform processing fields (such as deformation rates, pressure, temperature, extended surface area) which are best realized through miniaturization with controlled micro-architecture of the intensified reactor providing accessibility for heat $/ \mathrm{mass}$ transfer facilities. Secondly, (known as phenomenon based PI) PI results from an underlying inherent phenomenon which is only observed in the presence of enhanced processing field and/or miniaturization which in fact constitute the two prerequisite process conditions for PI.

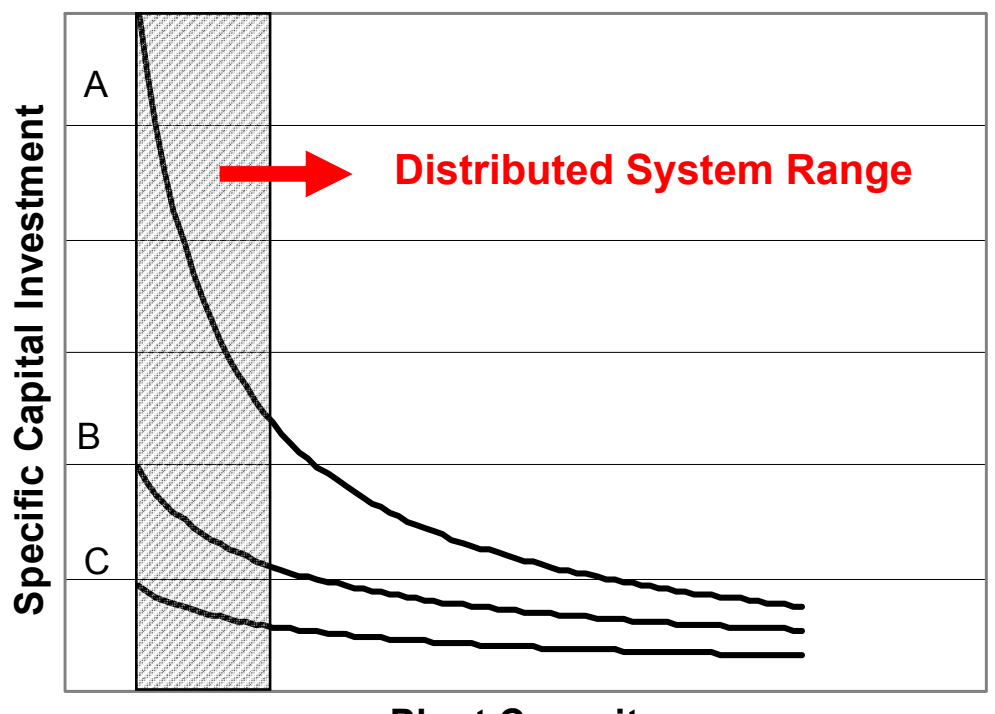

Plant Capacity

Figure 1: Variation of specific capital cost with plant capacity as a function of TAD: A: Current technology; TAD $=1 \mathrm{k} \mathrm{m} / \mathrm{m}^{3} ;$ B: Microcapillary reactor technology (Intensified Process) with TAD $=10 \mathrm{k}$ $\mathrm{m}^{2} / \mathrm{m}^{3}$; C: Micro-reactors using nano-structured micro-porous materials with applied intensification field (Intensified Hybrid Reactors) with TAD $=100 \mathrm{k}-1000 \mathrm{k} \mathrm{m} / \mathrm{m}^{3}$. 


\subsection{Importance of PI in sustainable chemical and energy production}

Phenomenon based PI, coupled with high throughput microreactor technology (Process Intensification and Miniaturization, PIM) is more powerful in delivering intensification above 10 fold or more; in some cases reaching 1000 fold compared with the existing technology. One of the effects of PIM is that, the specific capital cost of intensified processes has weak dependency on capacity. This technology does not have the burden of 'economies of scale', a defining characteristics commonly associated with large scale centralised production facilities such as refineries, chemical and power plants. It is possible to relate the specific capital cost of the centralised production plant with TAD (transfer area density which is area per unit volume for heat and/or mass transfer), of the reactors as shown in Figure 1 [2].

Figure 1 indicates why the current production plants must have large capacity in order to circumvent the burden of economies of scale. Intensified distributed production plants which consist of intensified unit operations do in fact deliver low specific capital and operating costs with short start up and shut down times. The distributed chemical and power generation through intensified integrated bio-refineries is essential for sustainability and in combating global warming using biomass and biomass waste as renewable feedstock instead of fossil fuels.

\subsection{Nano-structured micro-porous materials for PI}

Figure 1 also illustrates the importance of materials science, including the catalysis and reactor design to achieve PI in distributed production. By increasing the transfer area density for heat and mass transfer in reactors, capital cost is substantially reduced in distributed-intensified plants. Transfer area density can be enhanced by using monolithic high throughput micro-reactors in which the reaction media is made of nano-structured micro-porous materials. Nano-structure provides catalytic surface area while micro-porosity providing accessibility of the catalytic sites.

These materials can be made of polymeric, metallic or ceramic or in composite form fabricated into monolithic agro- bio- or chemical-reactors. It is also possible to add other process intensification fields to such reactors in order to achieve 'Phenomenon based process intensification'. Current examples of PI cover agro-processes, biotechnology, chemical and energy conversion processes $[1,3]$.

\subsection{Processing strategy in the human body}

The objective of this work is to utilize nature's (the human body) processing strategy in the design of micro-reactors for AgroProcess, BioProcess and Chemical Process Intensifications while designing micro-bioreactors for organ replacement in the form of transplants.

Due to its long evolution, we can assume that the human body is an intensified biochemical plant formed by the integration of unit operations in the form of organs and monitored and controlled by neural and hormonal systems. The macroscopic architecture of the plant is provided by the skeletal structure 
which itself interact with the organs and connective tissue. The important elements of the body's processing strategy can be summarised as:

1) High surface area-to-volume ratio for heat-mass transfer. For example, for human adult lungs TAD is ca. $10^{4} \mathrm{~m}^{2} / \mathrm{m}^{3}$.

2) Hierarchical length scale in organs involving flow such as lungs, liver and (millimeter; micro; nano connectivity) for simultaneous accessibility, continuous process and maintenance.

3) Mass transfer and communication (also quorum signalling by cells) across nano-size barriers (bilayers).

4) Monolithic unit operations with continuous product removal.

5) Physiological stress dependent response in 3-Dimensional cell culture with its own cell-support system.

The hierarchic length scale in the liver can be seen in Figure 2 [4].
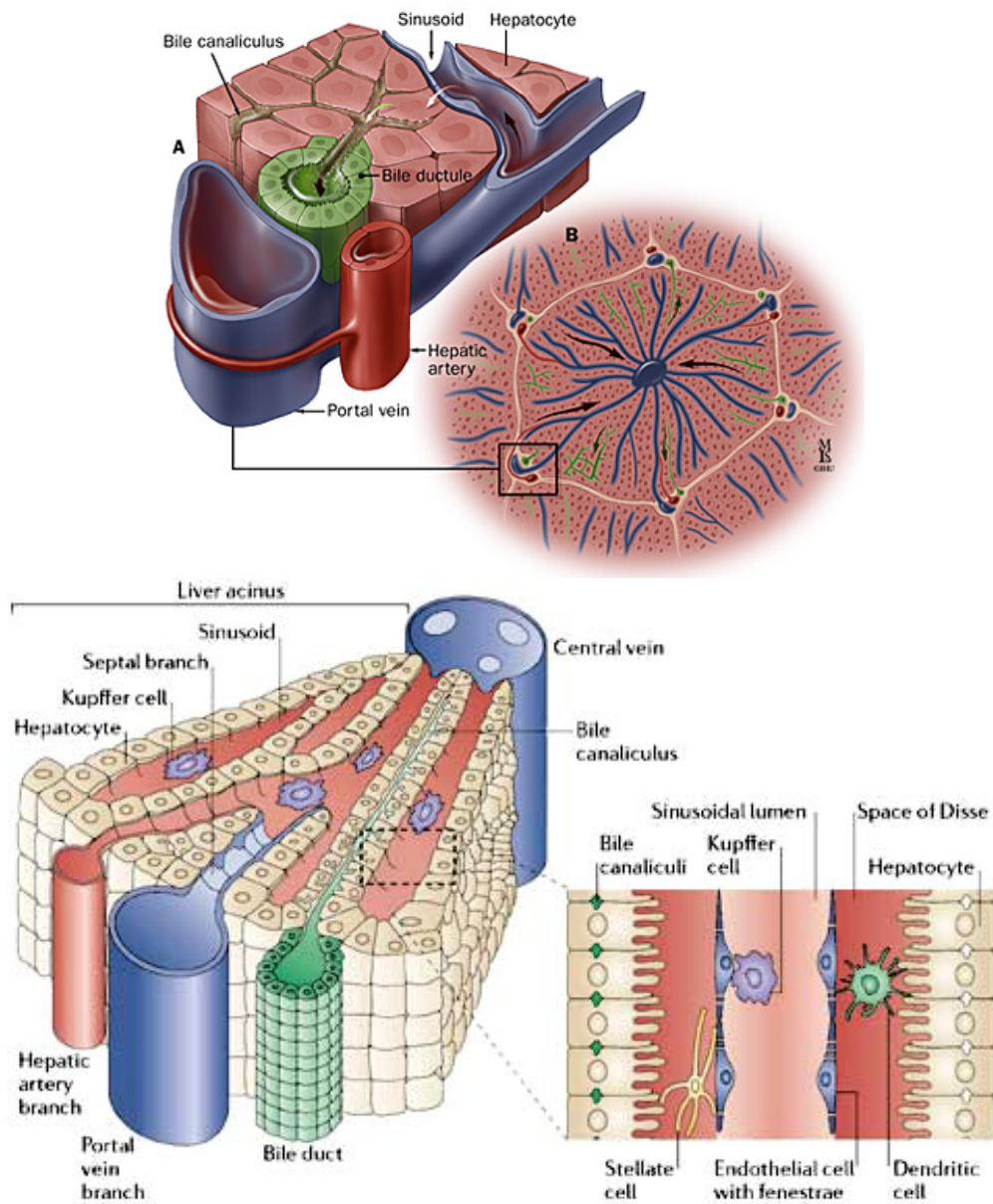

Figure 2: Macro- and micro-level structure of the liver showing the architecture of the unit operation. 
The liver receives a dual blood supply from the portal vein and hepatic artery which join in the capillary bed of the liver and exit via central veins, coalescing into hepatic veins, then leave the liver. Anatomically, based on the blood supply, the liver is divided into two 'hemilivers' which further divide into eight segments which focus upon subdivisions of the hepatic and portal veins. Each of these segments is in turn divided into lobules which centre on central portal veins. The liver is divided microscopically into functional units called 'acini'. These centre around a vascular stalk (terminal branches of the portal veins and hepatic arteries) and bile ducts and are comprised of the functioning cells of the liver (hepatocytes). These highly organised functional units allow optimal productivity of the liver whilst forming a compact structure, reflecting the processing strategy cited above.

\subsection{Significance of 3D culture and quorum signalling in BioProcess Intensification}

During bioprocessing, micro-organisms undergo physiological stress which alters their metabolism and physiology. It is therefore not surprising that a number of scale-up responses are observed when the performance of bacteria in small and large bioreactors is compared. These differences include, biomass yield, recombinant protein accumulation and cell viability [5]. Strong production of recombinant proteins interferes with cellular processes and the cells readjust metabolic fluxes and enzyme composition which may result in inhibition of growth or low level of product accumulation [6]. It is then possible to adopt production schemes that enhance volumetric productivity and sustainability of the process.

Recently, we have shown that bioprocesses could be intensified (Bioprocess Intensification) through the immobilization of bacteria within the micro-pores (within certain size range) of nano-structured micro-porous polymers which alter the metabolic activity of the bacteria [7]. This phenomenon is similar to that we observed for mammalian cells whereby the cell proliferation, protein production rate and differentiation are dependent on the size of the micro-environment that they grow [8-11]. The observed bioprocess intensification is over 30 fold compared with the best of the existing technology. As a result, for a given bacteria or animal cell, an optimum pore size is present in order to maximise productivity and proliferation. We have also observed that bacterial growth under maximum productivity is in monolayer form without the formation of biofilm which occurs when the pore size of the support was large.

Another factor for enhanced in vitro protein production during 3D cell culture (in bone or cartilage cells) using micro-bioreactors in the form of cell support, is the chemical environment of the cell growth media. Both bone cells and cartilage cell growth rates and marker protein productions are enhanced by several hundred percent when bio-responsive coatings (such as hydroxyapatite) are used. These results indicate that the chemical and physical structure of the micro-environment affect cell viability, productivity and differentiation as observed for bacteria. 
It is now well understood that the $3 \mathrm{D}$ in vitro culture of cells is essential in order to provide the necessary micro-architecture for the cell growth similar to that observed in nature. This micro-architecture provides cell-cell communication for collective function as cell complete autonomy will not result in tissue specificity. Isolated cells lose most functional differentiation when separated and placed in traditional cell cultures. There is now a growing opinion that cell function is regulated by microenvironment and tissue architecture [12]. The effect of micro-environment in bacteria is evaluated in terms of environmental stress as well as in terms of alteration of the metabolic pathway and it is suggested that the physiological stress should be controlled in order to control the metabolic activity, viability, differentiation and productivity, gene expression pattern including mRNA levels of molecular chaperons, proteases, lysis genes. However, in large scale fermentation reactors, or in supported bioreactors without a uniform or suitable pore micro-architecture and stressor field, the physiological response of the bacteria to the micro-environment is not collective due to the transient and variable nature of the stressor.. Therefore the phenomenon of micro-environment induced physiological response will be absent in the absence of quorum signalling.

\section{Experimental}

Experiments were conducted in 4 levels. Here, experimental details are not provided but we refer to our previous publications. They include: 1) Preperation of nano-structured micro-porous (NSMP) polymers [11, 13]; 2) NSMP-catalysts /catalyst support / monolithic reactors [14]; 3) in vitro bone growth [8-11].

\section{Results}

\subsection{Nano-structured micro-porous polymers for tissue engineering and monolithic bioreactors with 3D-culture}

As shown previously [1,7-11] the pore and interconnecting hole sized as well as the pore volume are important in the growth, viability and differentiation of cells in vitro. While the architecture of the support provides a 3D-culture and cell-cell communication, biochemical compatibility or even biochemical activation of the cells can be achieved by the chemical composition of the nanostructured micro-porous support material. The in-situ coating of the NSMPpolymers has shown to enhance bone and cartilage growth similar to those observed for naturally occurring peptide coatings [10]. In tissue engineering or bioprocess intensification when the pore structure is important, the pore and interconnect size distributions should be narrow. Figure 3 illustrates the pore structure of a typical NSMP-polymer. Fig 3(a) illustrates the pore and interconnect structures while Fig 3(b) shows the wall structure. Figs 3(c), (d) illustrate the nano-porosity of the pore surface and wall. 


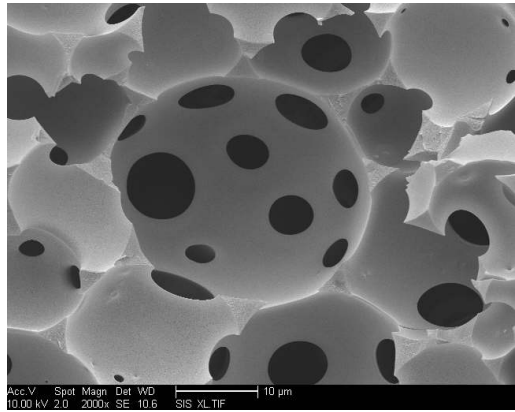

(a) Pore structure, Bar $10 \mu \mathrm{m}$

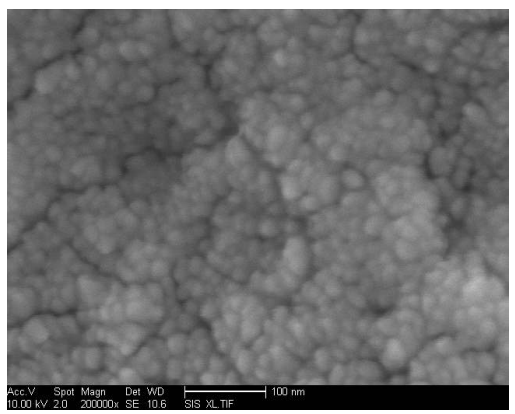

(c) Pore surface. Bar: $100 \mathrm{~nm}$

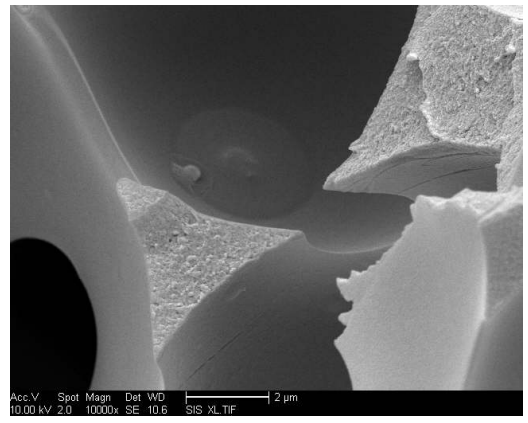

(b) Wall cross-section. Bar: $2 \mu \mathrm{m}$

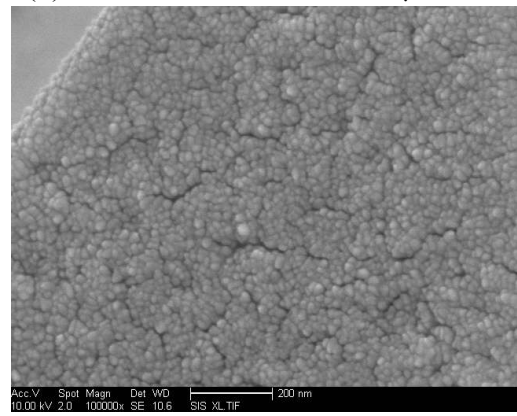

(d) Wall cross-section. Bar: $200 \mathrm{~nm}$

Figure 3: Typical NSMP-polymer structure suitable for monolithic bioreactors.

\subsection{Nano-structured micro-porous metals for intensified reactors}

Nano-structured micro-porous metals with structures very similar to those NSMP-polymers have been developed as catalyst, catalyst support for use as monolithic microreactors. Chemical processes have the advantage of being carried out at high or very low temperatures, pressures, concentrations, deformation rates and hence are more suitable for intensification. Further intensification can be achieved through the superimposition of a process intensification field (such as electric field) in hybrid reactors which often need to be monolithic. It is once again necessary to have a hierarchic pore structure so that the catalytic surfaces are accessible to the reactants. Figure 4 illustrate the hierarchy of the pores in a typical metallic monolith where the pore range can be over 4-5 decades. In these materials, pores are made from grains (size range $10 \mu \mathrm{m}-0.1 \mu \mathrm{m})$ as seen in Figures 4(a,b). The grain surface have nano-porosity (Fig 4 (c)) which can be controlled (typically 100-10 nm). Inside the grains, pore volume and pore size can be variable as shown in Fig 4(d) where the pore size is illustrated in Fig. 4(e). The structure of the NSMP-metals can be varied depending on the method of manufacture and its composition [13,14]. A variant structure is illustrated in Fig. 4(f). 


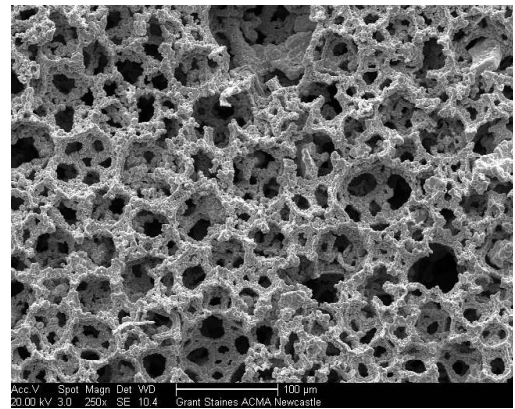

(a) General appearance (Bar: $100 \mu \mathrm{m})$

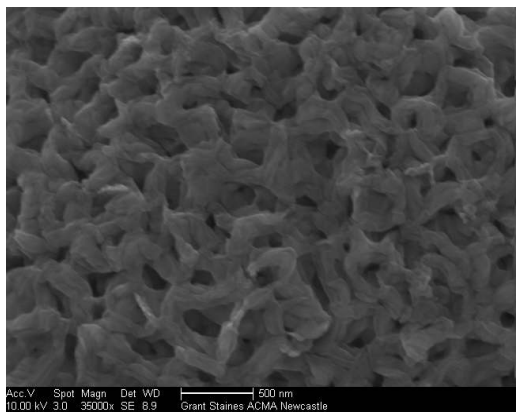

c) Grain surface (Bar: $500 \mathrm{~nm})$

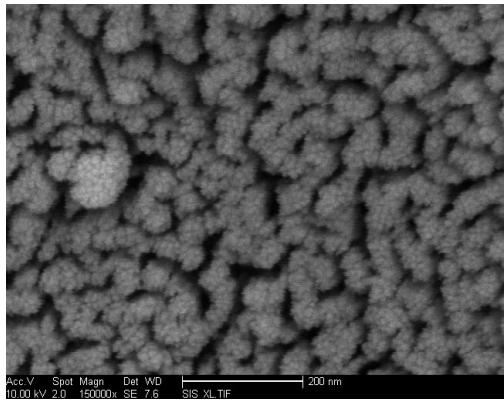

e) Detail of the inner grains (Bar: $200 \mathrm{~nm}$ )

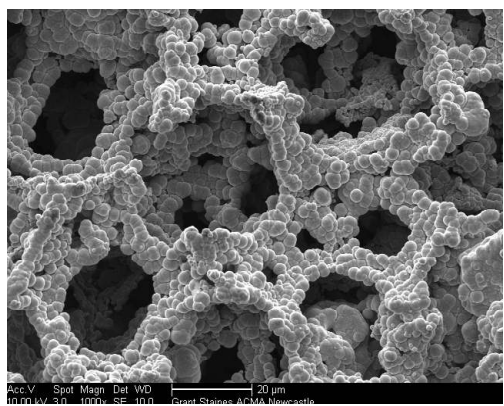

b) Wall structure (grains) (Bar: $20 \mu \mathrm{m})$

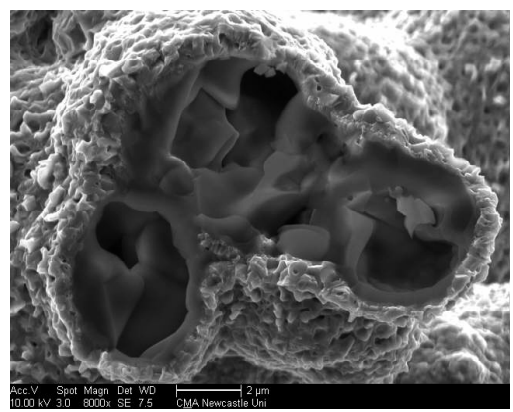

d) Inner structure of the grains (Bar: $2 \mu \mathrm{m})$

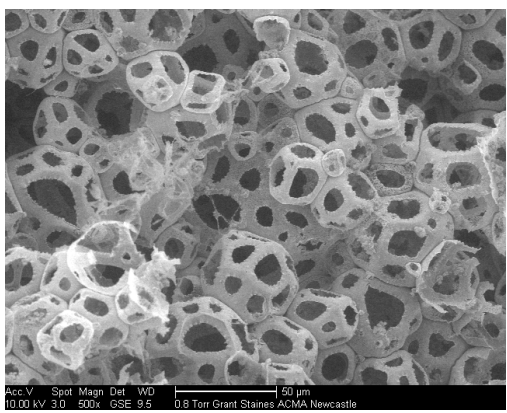

f) A variant of pore structure (Bar: $50 \mu \mathrm{m})$

Figure 4: Nano-structured micro-porous metal alloys with a hierarchy of pores.

\subsection{Bone tissue engineering}

The purpose of tissue engineering is to create artificial organs for in vitro testing or for transplant after growing of the tissue in vitro. It is also possible to graft the well characterised and optimised in vitro support. Here we optimise the chemical and physical structure of the support material for grafting. We used NSMP-polymers which were coated with hydroxyapatite. Figure 5 clearly shows 


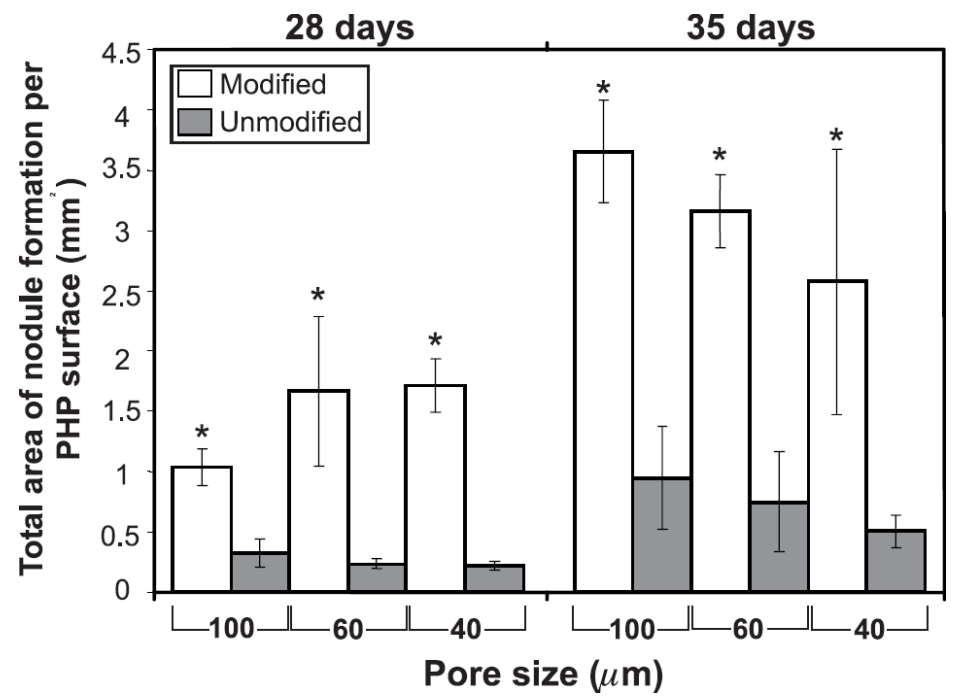

Figure 5: The effect of NSMP-polymer support pore size $(\mathrm{D}=100,60,40$ $\mu \mathrm{m})$ on mineralized nodule formation of rat osteoblast cells cultured in vitro after 28 or 35 days using hydroxyapatite coated (modified) or uncoated (unmodified) styrene NSMP-polymer (PolyHIPE Polymer) cell supports.

that the coating of the polymer increases mineralized nodule formation of rat osteoblast cells cultured in vitro and that $100 \mu \mathrm{m}$ pore support is more effective.

\subsection{Bone graft}

Repair of bone defects created by surgery, tumours, trauma, infections, and implant revisions may be enhanced by bone graft substitutes. Here we use NSMP-polymers also known as PolyHIPE Polymer (PHP) with pore size of 100 or $40 \mu \mathrm{m}$. In our previous studies PHP has been found to be biocompatible with at several different cell types such as fibroblasts, osteoblasts, macrophages and chondrocytes. Biocompatibility studies demonstrated that there was no cytotoxic effect observed when cells were cultured within PHP in in vitro. The purpose of this investigation was to determine whether PHP is biocompatible and if the incorporation of HA in PHP scaffold would enhance the biological properties of living body response as compared to different pore sized PHP scaffolds. Extracellular matrix (ECM) production was examined by histological analysis and indicated new bone had been formed in PHP. Graft area morphology was also examined using environmental scanning electron microscopy (ESEM). The inflammatory response to PHP was negligible at both short (4 weeks) and long (12 weeks) time points. These results indicate that the addition of hydroxyapatite in PHP improves osteoblast response as compared PHP without hydroxyapatite. All PHP implants were tolerated; no necrosis or abscess formation was observed around either type of implants. Figure 6 shows the implant after 12 weeks within 
the frontal section of rabbit iliac bone showing good integration between the implant (PHP) and bone (B). In this scanning electron micrograph, the implant is $100 \mu \mathrm{m}$ pore size PHP coated with hydroxyapatite. In Figure 6(a) the connectivity of the bone and PHP implant is illustrated while Fig 6(b) shows osteoblast cell process (arrow) is present in the form of fibrillar collagen synthesized and organized by osteoblast. Heamatoxylin and Eosin (H\&E) stained
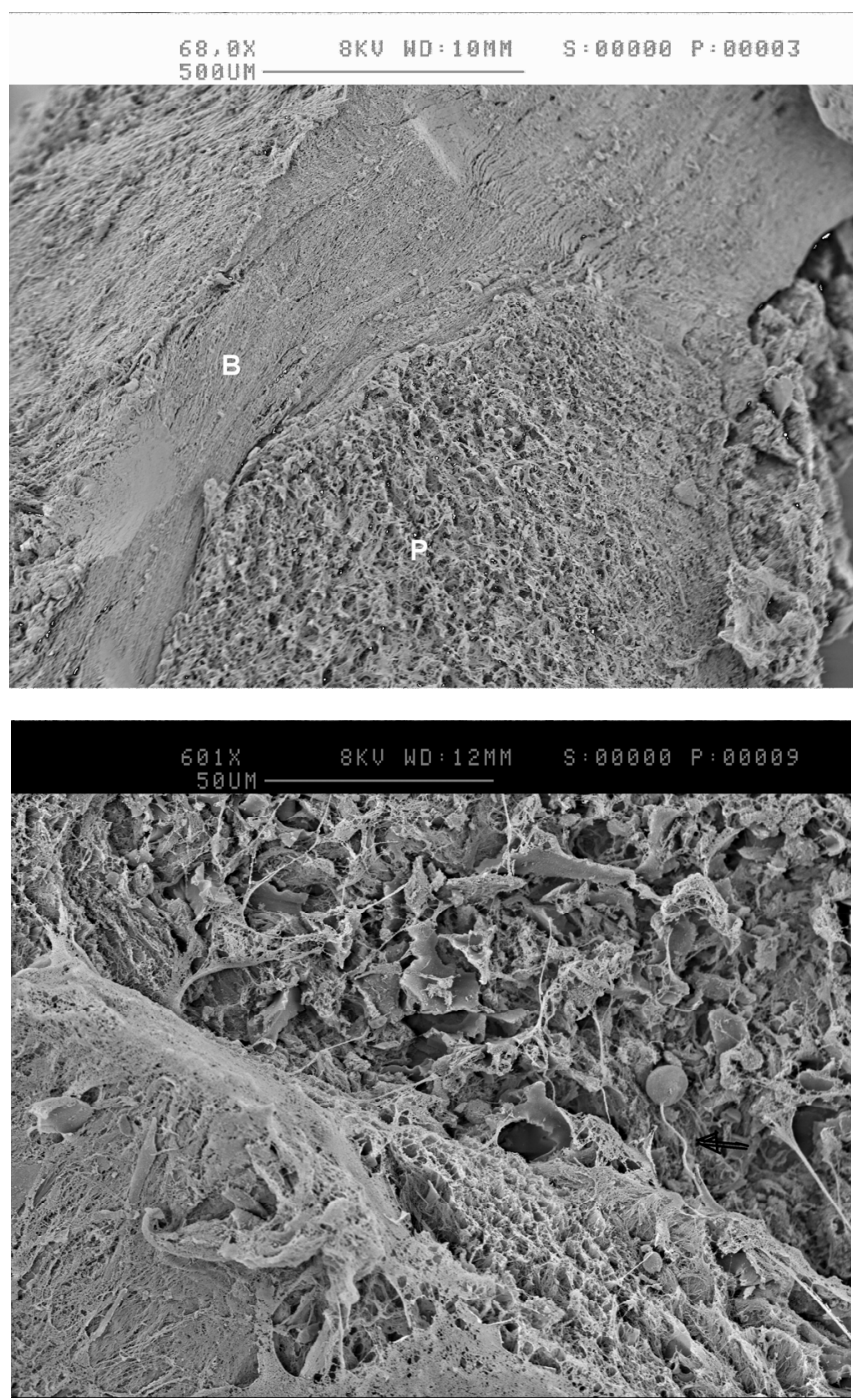

Figure 6: Scanning electron micrographs of bone implant showing: (a) their connectivity; (b) presence of osteoblast cell process (arrow) in the implant. 
histological section of $40 \mu \mathrm{m}$ hydroxyapatite coated PHP implant show the presence of vascularisation indicating that the implant was fully integrated into the body.

\subsection{Model in vitro organ and intensified monolithic reactors}

Figure 7 is a diagrammatic illustration of an intensified monolictic micro-reactor which can be used for biological or chemical conversion processes. It is made from nano-structured micro-porous materials, either polymeric (structure described in Figure 4) or metallic (structure described in Figure 5). The bulk of the monolith provides the surface area for bio- or chemical catalysis while the array of large (millimeter size) channels provide heat and mass transfer facility close the catalytic reaction sites. This reactor is very similar to the liver structure described in Figure 2 and hence can be used as a model for the liver. The porosity of the interface between the large channels and the micro-porous bulk can be controlled. When the interface is non-porous, pressures in the channels and the bulk can be controlled independently.

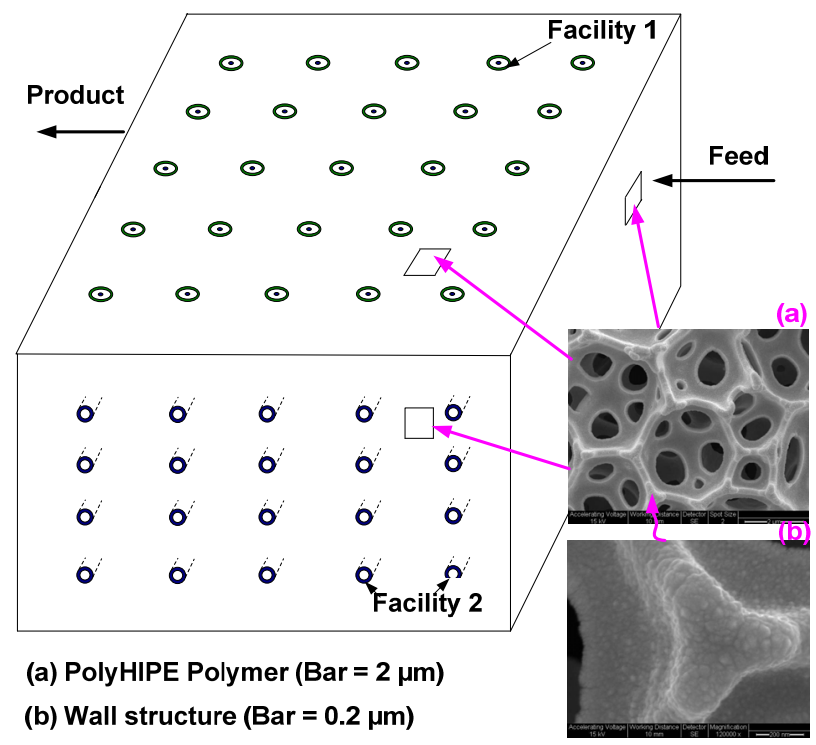

Figure 7: A model for an in-vitro organ or intensified micro-bioreactor.

\section{References}

[1] Akay, G., Bioprocess and chemical process intensification. In: Encyclopedia of Chemical Processing, Ed: S Lee, Marcel Dekker, NY. pp. 185-198, 2006.

[2] Akay, G., Renewable resources come together, The Chemical Engineer, 784, 27-30, 2006. 
[3] Akay, G., Burke, R.D., Synthetic symbiosis system as soil additives to deliver active ingredients through plant roots for enhanced plant and crop yield. PCT Patent Application PCT/GB09/02380, 2009. Also: J. Agricultural Sci., (accepted for publication).

[4] Adams, D.H. \& Eksteen, B., Aberrant homing of mucosal T cells and extraintestinal manifestations of inflammatory bowel disease, Nature Reviews Immunology, 6, 244-251, 2006.

[5] Enfors, S-O., et al., Physiological responses to mixing in large scale bioreactors, J. Biotechnology, 85, 175-185, 2001.

[6] Hoffman. F., \& Rinas, U., Stress induced by recombinant protein production in Escherichia coli, Adv Biochem Engin/Biotechnol., 89, 73-92, 2004.

[7] Akay, G., Erhan, E., Keskinler, B., Bioprocess intensification in flow through micro-reactors with immobilized bacteria, Bioengineering Biotechnology, 90, 180-190, 2005.

[8] Bokhari, M. Birch M., and Akay, G., Polyhipe polymer: A novel scaffold for in vitro bone tissue engineering, Advances in Experimental Medicine and Biology, 534 , 247 - 254 (2003).

[9] Akay, G., Birch, M.A., Bokhari, M.A., Microcellular Polyhipe polymer (PHP) supports osteoblastic growth and bone formation in vitro, Biomaterials, 25 3991-4000, 2005.

[10] Bokhari, M.A., Akay, G., Zhang S., and Birch, M.A., A hybrid biomaterial combining the peptide hydrogel RAD 16-1 with PolyHIPE Polymer (PHP) enhances osteoblast growth and differentiation in vitro. Biomaterials, 26 , 5198-5208, 2005.

[11] Akay, G., Bokhari, M.A., Byron, V.J., and Dogru, M., Development of nano-structured materials and their application in bioprocess-chemical process intensification and tissue engineering. In: Chemical Engineering Trends and Developments, Ed: MA Galan and E.M. Del Valle, Wiley, London, pp. 171-196, 2005.

[12] Bissell, M.A., Rizki, A., Mian, I.S., Tissue architecture: the ultimate regulator of breast epithelial function, Curr. Opin. Cell Biol., 15, 753-762, 2003.

[13] Akay. G., Dawnes, S., Price, V.J., Microcellular polymers as cell growth media and novel polymers, EP 1183328, 2002.

[14] Akay, G., Calkan, B., Hasan. H., and Mohamed R., Preparation of nanostructured microporous composite foams, PCT/GB09/02403, 2009. 